\title{
IL REFERENDUM DEL REGNO UNITO SULLA BREXIT ED IL SUO IMPATTO SUL MERCOSUL NELLA PROSPETTIVA DEI DIRITTI SOCIALI
}

\author{
Francesco Bilancia \\ Professor de Direito Constitucional - Università “G. d'Annunzio” di Chieti - Pescara/Italia.
}

\section{Sandra Regina Martini}

Professora do Centro Universitário Ritter dos Reis (UniRitter - Canoas, RS). Professora visitante do Programa de Pós-Graduação em Direito da Universidade Federal do Rio Grande do Sul (Porto Alegre, RS). E-mail: <srmvial@terra.com.br>.

\begin{abstract}
Prendendo le mosse dalle tematizzazioni polemiche professate durante la campagna referendaria svoltasi nel Regno Unito per il voto del giugno 2016 sulla Brexit, il saggio apre una riflessione critica sulle trasformazioni da tempo in atto, nel diritto dell'UE e nella giurisprudenza della Corte di giustizia così come in alcuni ordinamenti degli Stati membri, in relazione ai diritti alle prestazioni sociali dei cittadini europei residenti in Paesi membri diversi dal proprio ed al possibile impatto di tale fenomeno su altri sistemi integrati di mercato come il Mercosul. II quadro che ne emerge induce a più approfondite riflessioni sulle cause della crisi di legittimazione attuale dei sistemi di integrazione politica ed economica, come il processo di integrazione europea, il cui cedimento è gravemente accelerato dalla messa in discussione dei diritti sociali in danno di una più forte nozione di cittadinanza europea; e come in altre realtà istituzionali e sociali, in particolare nel Mercosul.
\end{abstract}

Keywords: Brexit. Mercosul. Diritti sociali. Unione Europea.

Sommario: 1 Una premessa. II diritto nella società contemporanea - 2 II referendum sulla Brexit e i suoi riflessi sul Mercosul - $\mathbf{3}$ II tema dell'immigrazione nella campagna referendaria UK: un problema futuro o già attuale peril Mercosul? - 4 Considerazioni finali - Bibliografia

\section{Una premessa. II diritto nella società contemporanea}

L'accesso universale degli individui alle prestazioni dei diritti sociali ha rappresentato una delle prerogative degli sviluppi degli ordinamenti nazionali nel corso del secolo scorso, mentre la sfida di questo secolo è rappresentata dall'implementazione di tali diritti. Ciò ha comportato, per le analisi condotte 
dalle scienze sociali, la presa in esame delle implicazioni di tali diritti anche nella Ioro declinazione in termini di doveri, responsabilità, decisioni dei pubblici poteri e adattamenti delle organizzazioni sociali. Oggi non è più possibile negare la positivizzazione giuridica dei diritti sociali (nonostante essi vengano costantemente violati); tuttavia emergono con forza altre questioni, spesso di carattere materiale. Si pensi ad esempio alla portata della estensione del diritto alla salute: è materialmente possibile pensare che tutti gli esseri umani possano realmente beneficiare delle relative prestazioni? In altra prospettiva, poi, gli individui potrebbero non rivendicare l'accesso a particolari cure; oppure potrebbero rifiutarsi di esporre al personale sanitario i loro disturbi, i problemi propri o dei propri familiari. Quale che sia la prospettiva di analisi prescelta, quindi, la discussione sulla natura, portata e struttura dei diritti fondamentali pur avendo effetti fortemente positivi sugli sviluppi della civiltà giuridica e politica e sull'evoluzione dei sistemi sociali, potrebbe non apparire affatto risolutiva.

Altra importante dimensione problematica: siamo davvero convinti che tutti desiderino che tali diritti vengano effettivamente implementati? Non è possibile, infatti, nascondere che tra le conseguenze del loro riconoscimento si annoverino rilevanti conseguenze in termini di doveri, responsabilità, conflitti tra situazioni soggettive e garanzie di attitudini alla reciprocità. Se, ad esempio, partiamo dal presupposto che il diritto alla vita implichi che...si viva, ci si dovrà pur interrogare sulle condizioni materiali di questa vita. Così, se soffro di qualche malattia incurabile e non desidero più soffrire, qual è il diritto fondamentale che meriterà di prevalere? Oppure, se ho un problema di salute e non desidero renderlo noto a nessuno, è davvero indispensabile che io mi curi? E, ancora ad esempio, in relazione la mio orientamento sessuale, esiste il diritto ad essere come desidero? E se la mia malattia comporta problemi per la comunità? Quale tra i tanti diritti in potenziale conflitto deve essere ritenuto prevalente? II diritto alla libertà nella sua forma più amplia, come right to be let alone, non è forse anch'esso un diritto umano? Chi ne può essere davvero titolare? Nella prospettiva problematica che si sta evocando tanto il diritto alla vita quanto il diritto alla salute rischiano di essere rappresentati all'opposto come veri e propri doveri, e se assumiamo come vero che il diritto alla vita implichi il diritto di non soffrire, si rende di immediata evidenza il suo carattere di paradosso. La società contemporanea presenta nella realtà esperienziale moltissimi paradossi di questo tipo, paradossi che si traducono in conflitti tra diritti fondamentali e tra interessi contrapposti. Conflitti e contrapposizioni di interessi che si manifestano, inoltre, in una società in cui i confini politici e geografici hanno un'importanza sempre minore. Viviamo in effetti in una società-mondo, circostanza che rende poi non tanto paradossale che ci 
si possa interrogare sui possibili effettiche un fenomeno quale quello della c.d. BREXIT possa avere sul sistema del Mercosul.

Nella prospettiva di analisi contemporanea, ad esempio, uno spazio sempre più ampio viene assunto dalla questione del costo dei diritti sociali in funzione della loro universalità. In tesi generale è possibile sostenere che il loro mancato riconoscimento in quanto tali, vale a dire in quanto diritti universali, comporti un costo sociale e politico molto elevato; ma il fatto che essi non vengano effettivamente garantiti implica un costo ancora maggiore, senz'altro nella prospettiva economica. Mettere a tema la questione dei diritti fondamentali nel contesto sovrastatuale apre, inoltre, la riflessione sui confini della "autonomia" individuale: se collochiamo l'individuo in un contesto relazionale in cui la sua soggettività viene incastrata in un sistema di connessione interindividuali secondo lo schema diritto/dovere è evidente che si riduce la sfera di autonomia del singolo. Siamo sicuri di voler realmente essere autonomi? Come rendere tale autonomia compatibile con le rivendicazioni dei diritti e con le conseguenti attivazioni delle relazioni formali diritti/doveri? Si tratta indubbiamente di questioni teoriche che rendono necessaria una riflessione. Una riflessione che si ampia e si complica nella prospettiva di considerare tali questioni oltre i confini statali, in ambiti istituzionali ed ordinamentali composti come luoghi di integrazione economica. ${ }^{1}$ Le organizzazioni sovrastatali dei mercati a prevalente impianto di integrazione economica, possono rappresentare infatti un "locus" anche per la realizzazione dei diritti sociali, ${ }^{2}$ anche assumendo la prevalente vocazione economica di tali sistemi istituzionali.

La prospettiva di riflessione che qui si intende promuovere pretende di orientare lo sguardo oltre gli avvenimenti più recenti, al fine di cogliere le tracce che da singoli eventi si mostrino in realtà come elementi costanti di evoluzioni in atto contemporaneamente in sistemi apparentemente molto diversi e molto lontani tra loro. Ma la proposta di analisi ci è apparsa sensata. E' la ragione per cui questo saggio assume come obiettivo quello di avviare una discussione che prenda le

1 Sul tema si veda JAEGER JÚNIOR, Augusto; DIZ, Jamile Bergamaschine Mata. Por uma teoria jurídica da integração regional: a inter-relação direito interno, direito internacional público e direito da integração. Revista de Direito Internacional, Brasília, v. 12, n. 2, p. 138-158, 2015. p. 156: “[...] necessidade de estabelecer os pressupostos para a construção de uma teoria própria e autônoma que pudesse explicar a consequente evolução e desenvolvimento, mesmo que fragmentado, desse tipo de associação interestatal" ("necessità di stabilire i presupposti per la costruzione di una teoria propria e autonoma che potrebbe spiegare l'evoluzione conseguente e lo sviluppo, come anche la frammentazione, di questo tipo di associazione interstatale").

2 In questo senso, Roberto Ruíz Díaz Labrano fa notare che i processi di integrazione riguardano gli ambiti giuridico, politico e sociale (DÍAZ LABRANO, Roberto Ruíz. La integración y las constituiciones nacionales de los Estados Parte del Mercosur. In: KONRAD ADENAUER STIFTUNG. Anuario de Derecho Constitucional. Buenos Aires: CIEDLA, Konrad-Adenauer-Stiftung, 1999. p. 65-78). 
mosse dal processo drammatico che sta compromettendo il futuro dell'Unione europea per come avevamo imparato a considerarla, perché le conseguenze che tale processo produrrà sulla garanzia dei diritti sociali in ambito europeo avranno un significativo impatto anche nel contesto globale, in particolare nei Paesi del Mercosul. Sebbene le analisi in relazione a tali connessioni siano finora piuttosto scarse ${ }^{3}$ riteniamo sia fondamentale ragionare in prospettiva comparativa, nella consapevolezza che eventi importanti che pur abbiano luogo in un punto specifico del mondo abbiano effetti diretti sulla c.d. "società globale".

Riflettere sul significato del diritto ${ }^{4}$ oggi comporta la riconsiderazione del carattere più tradizionale della sua diomensione storica, quello dei limiti territoriali di efficacia, laddove all'opposto nella società contemporanea "frontiere e barriere" 5 rappresentano pur certo tuttora un limite, ma allo stesso tempo la tensione originale dello sviluppo della dimensione del comunque possibile. Se la tradizione statalista ha utilizzato le frontiere come occasione di guerra, o come strumento che rendesse possibile e proficuo, ad esempio, lo sfruttamento sessuale o le azioni della criminalità organizzata, oggi sappiamo che oltre le frontiere statali è invece possibile un altro tipo di vita, un'altra congerie di esperienze.

Ebbene, il recente referendum del Regno Unito sulla c.d. Brexit presenta analoghe contraddizioni, analoghe incertezze. Rappresenta in effetti una nuova, imprevista ipotesi di manifestazione della richiamata dicotomia limite/possibilità, anche in riferimento alle trasformazioni in atto del fenomeno giuridico che si assume produrranno rilevanti effettianche in altri luoghi del pianeta, come ad esempio nel sistema del Mercosul. La costituzione del sistema del Mercosul è molto più recente dell'Unione europea, eppure non è insensato riflettere in una prospettiva di società globale sui possibili effetti delle trasformazioni che subiranno, ad esempio, le garanzie dei diritti sociali a seguito del voto del giugno 2016 nel Regno Unito. E' noto che la più gran parte degli analisti si sia soffermata sui riflessi del referendum nel sistema della UE, e soprattutto nel Regno Unito, dove già si manifestano intenti di adozione di misure normative restrittive dei diritti degli Europei non cittadini. Addirittura un recente attentato contro cittadini di

3 Nelle ricerche realizzate su questo tema non ci siamo imbattuti in articoli che si concentrassero in maniera specifica sugli effetti della c.d. BREXIT nel Mercosul, malgrado siano invece molti quelli dedicati agli effetti sull'Unione Europea. C'è evidenza addirittura di alcune pubblicazioni sugli effetti di tale processo in Cina, pur sempre in riferimento a questioni economiche.

4 Il diritto non sta nei concetti ma nella comunità, nei gruppi, nell'esercizio concreto del potere e dell'influenza della religione, nella vita economica, nell'opinione pubblica.

5 Sul tema si veda l'interessante studio di GEUSS, Raymond. Beni pubblici bene privato: origine e significato di una distinzione. Roma: Donzelli Editore, 2005. p. 41: “L'esistenza della barriera stessa costituisce parte dell'attrattiva e del piacere che si prova nel superarla". 
religione islamica ${ }^{6}$ è stato messo in relazione alle tensioni scaturite all'esito del referendum. Nel caso del Mercosul, non è al momento immaginabile l'ideazione di un referendum simile a quello celebratosi nel Regno Unito da parte di uno degli Stati membri, ${ }^{7}$ ma esistono strumenti che permettono di "espellere o sospendere" singoli Stati dal Mercosul in caso di scelte politiche che siano lette come violazioni gravi ai principi democratici.

Se si volesse procedere ad una analisi comparativa dei due sistemi di integrazione regionale, quello dell'Unione europea e quello del Mercosul, è indubbio che si rileverebbero, come è noto, profonde differenze tanto in relazione alle rispettive strutture giuridiche quanto dal punto di vista istituzionale. Alcune di queste differenze sono in effetti rilevanti proprio al fine di ipotizzare una valutazione circa gli effetti della Brexit nei due diversi sistemi. La prima ovvia differenza è data dal carattere sovranazionale del sistema dell'Unione Europea, laddove il Mercosul ha mantenuto una struttura essenzialmente intergovernativa. II primo sistema, a vocazione regionale, quindi si basa sull'attribuzione/distribuzione di competenze tra Stati membri ed istituzioni comuni, implicando tale distribuzione un permanente trasferimento di funzioni sovrane da parte degli Stati membri ad una organizzazione internazionale. ${ }^{8} \mathrm{Nel}$ secondo caso, invece, tutti i processi decisionali dipendono dall'assunzione di decisioni da parte di ciascuno degli Stati partecipanti. ${ }^{9}$

6 Si veda l'attentato di Finsbury Park a Londra, in cui il conducente di un van ha investito un gruppo di persone in uscita da una moschea (FINSBURY Park attack: what we know so far. BBC, 19 jun. 2017. Disponivel em: <http://www.bbc.com/news/uk-40323769>. Acesso em: 27 jun. 2017).

7 Sulla possibilità di un referendum nel Mercosul, si vedano i seguenti documenti: Tratado de Assunção (Decreto no 1.901, de 09 de maio de 1996); Tratado para a Constituição de um Mercado Comum entre a República Argentina, a República Federativa do Brasil, a República do Paraguai e a República Oriental do Uruguai (Tratado Mercosul, decreto no 350, de 21 de novembro de 1991); Protocolo Constitutivo do Parlamento Do Mercosul; Protocolo de Olivos para a Solução de Controvérsias no Mercosul (Decreto nํㅜ 4.982, de 9 de fevereiro de 2004).

8 Su questo tema Odete Maria Oliveira scrive che: “A supranacionalidade encontra-se envolvida em -torno de um conceito de natureza dinâmica e contornos difusos [...] observando sempre uma vinculação cumulada de três elementos essenciais na configuração dessa categoria: a independência das instituições comunitárias frente aos Estados-membros; a existência de relações diretas entre as instituições comunitárias e os particulares e a transferência de competências dos Estados em favor da unidade supranacional" ("La sovranazionalità è centrata su un concetto di natura dinamica e a contorni diffusi [...] si osserva sempre una combinazione che collega tre elementi essenziali nella configurazione di questa categoria: l'indipendenza delle istituzioni comunitarie nei confronti di ogni Stato membro; l'esistenza di rapporti diretti tra le istituzioni comunitarie e le istituzioni nazionali, ed il trasferimento di competenze statali a favore dell'unità sovranazionale") (OLIVEIRA, Odete Maria. União Europeia: processos de integração e mutação. Curitiba: Juruá, 2001. p. 68).

9 Cfr. sul tema: Martha Lucía Olivar Jimenez: “La concepción del Derecho Comunitario como un orden jurídico nuevo, com características próprias que lo diferencian del Derecho Internacional clásico y del derecho interno de los Estados mimembros, obedeció a la necessidad percebida por la doctrina y por la Corte de Justicia de crear mecanismos que aseguran el cumplimento de las obligaciones contraídas por los Estados en los tratados constitutivos" ("La concezione del diritto comunitario come un ordine giuridico nuovo, con caratteristiche proprie che lo differenziano dal diritto internazionale classico e dal diritto interno degli Stati membri, risponde alla necessità, percepita dalla dottrina e dalla Corte di giustizia, 
Se si procedesse, quindi, ad una classificazione di tali ordinamenti secondo l'impianto metodologico classico delle scienze giuridiche, mentre l'Unione europea viene qualificato come ordinamento "comunitario", il secondo si caratterizza per un processo di integrazione vincolato dalle regole proprie del diritto pubblico internazionale ${ }^{10}$ classico. Da ciò le tre caratteristiche fondamentali che la dottrina indica come proprie del diritto comunitario: la specificità del nuovo ordine instaurato; la prevalenza del suo sistema normativo sugli ordini giuridici interni; e l'effetto diretto di molte delle sue disposizioni normative. ${ }^{11}$ In questo contesto ordinamentale, proprio dell'Unione Europea, hanno potuto essere instaurate le libertà di circolazione dei lavoratori, delle merci, dei servizi e dei capitali, seppur in funzione della costruzione di un mercato interno, arrivando a poter garantire entro certi limiti addirittura il divieto di discriminazione nella fruizione delle prestazioni sociali ${ }^{12}$ ai cittadini dell'Unione europea residenti in uno Stato membro a prescindere dalla propria nazionalità. Nel Mercosul, nonostante le relazioni tra Stati siano anch'esse caratterizzate dalla prevalenza degli obiettivi di integrazione economica di mercato, e malgrado la particolare fragilità istituzionale e giuridica, ${ }^{13}$ è possibile affermare che i diritti sociali siano invece inclusi nel quadro normativo comune. ${ }^{14}$

I citati caratteri propri del sistema Mercosul divengono, quindi, essenziali al fine di tentare una valutazione delle possibili conseguenze di determinati eventi di portata regionale che possano però produrre significativi effetti sul piano

di creare meccanismi che assicurino il rispetto degli obblighi contratti dagli Stati nei trattati istitutivi") (OLIVAR JIMENEZ, Martha Lucía. La compreensión de la noción de derecho comunitario para una verdadera integración en el Cono Sur. In: BASSO, Maristela (Org.). Mercosul: seus efeitos jurídicos, econômicos e poíticos nos estados-membros. Porto Alegre: Livraria do Advogado, 1997. p. 51).

10 JAEGER JUNIOR, Augusto. Metodologia jurídica europeia e mercosulista: considerações fundamentais. Revista da Secretaria do Tribunal Permanente de Revisão do Mercosul, Assunção, n. 3, mar. 2014. p. 121.

11 OLIVAR JIMENEZ, Martha Lucía. La compreensión de la noción de derecho comunitario para una verdadera integración en el Cono Sur. In: BASSO, Maristela (Org.). Mercosul: seus efeitos jurídicos, econômicos e poíticos nos estados-membros. Porto Alegre: Livraria do Advogado, 1997. p. 35-48.

12 Secondo Michel Ayral: "Il y a cependant, dans le mécanisme d'integratión de la communauté, des particularités qui en font un mécanism sui generis, reposant sur un processus dynamique d'harmonization des systémes nationaux pour éviter que la libre circulatión dans l'espace ainsi integré ne mette en cause des préocupations légitimes des pouvoirs publics, des enterprises ou des citoyens" ("Vi sono, tuttavia, nei meccanismi di integrazione comunitari, caratteristiche particolari che lo rendono un meccanismo sui generis, basato su un processo dinamico di armonizzazione dei sistemi nazionali per evitare che la libera circolazione nello spazio in tal modo integrato non metta in discussione le legittime preoccupazioni di governi, imprese e cittadini") (AYRAL, Michel. Le marché intérieur de l'Union européenne: les règles du jeu. Paris: Le Documentation Française, 1998. p. 7).

13 JAEGER JUNIOR, Augusto. Metodologia jurídica europeia e mercosulista: considerações fundamentais. Revista da Secretaria do Tribunal Permanente de Revisão do Mercosul, Assunção, n. 3, mar. 2014. p. 126.

14 SOARES FILHO, José. Mercosul: surgimento, estrutura, direitos sociais, relação com a Unasul, perspectivas de sua evolução. Revista CEJ, Brasília, ano XIII, n. 46, jul./set. 2009. p. 21-38. 
internazionale - come nel caso della Brexit -. A differenza del sistema dell'Unione Europea, infatti, qui la concretizzazione di qualsiasi misura normativa adottata in ambito regionale dipende dai processi decisionali interni agli ordinamenti degli Stati membri.

Questi elementi non possono non assumere un peso specifico rilevante qualora ci si accinga a la considerare le spinte mosse dalla necessità costante di oltrepassare i confini nazionali che, in molti casi, non sono neanche segnati dalla natura, ma dalle forme storiche assunte dagli ordinamenti statali tuttora fondati sull'intenzione discriminatoria tra cittadini e stranieri, con ciò trascinando una significativa quota di disuguaglianza sociale. I confini sono serviti a lungo a separare e a dividere, è nella loro stessa natura. Oggi, però, le pressioni sociali sono quasi inarrestabili quanto alla pretesa di oltrepassarli, quasi a pensare alla totale assenza di soluzioni di continuità territoriale. Ma la politica ed il diritto, oltre la dimensione sociale, non riescono ancora a metabolizzare questa prospettiva di unificazione: quel che tende a manifestarsi nel sociale, richiedendo il superamento di tali limiti, non trova ancora riscontro in un'adeguata risposta giuspolitica da parte degli ordinamenti degli Stati nazionali. Anci, nella ricerca costante di possibili percorsi di superamento, spesso si assiste addirittura alla costruzione di ulteriori limiti, come ad esempio quelli ascrivibili alla burocrazia, limiti formali che ostacolano ancora di più la libera circolazione delle persone, e l'arenarsi della spinta ideale di principi quali quelli di solidarietà e fraternità. Principi che, oltre la loro dimensione utopica, manifestano invece tutta la loro plausibilità proprio al cospetto della crisi dello Stato-nazione ed alla necessità di consolidare una società cosmopolita in cui l'umanità possa affrancarsi dai rischi generati dalle minacce che già aziona di fronte a sè stessa. ${ }^{15}$

Di fronte a tali minacce per l'umanità, il movimento pro-Brexit ha ritenuto di trovare una risposta nella ipotesi di un maggiore controllo sulle migrazioni verso il Regno Unito sostenendo che l'immigrazione possa danneggiare i cittadini del Regno Unito in termini di posti di lavoro, salari e qualità della vita, in considerazione della costante crescita dei flussi migratori negli ultimi vent'anni, soprattutto di provenienza da Paesi della stessa Unione Europea. ${ }^{16}$

15 “L'umanità è come l'ecologia, che non è fatta soltanto di fiumi incontaminati e di aria pulita ma anche del Ioro contrario: I'umanità, si diceva, si può minacciare soltanto da se stessa. II suo paradosso sta tutto in questa sua dimesione ecologica; così i diritti inviolabili dell'umanità non possono che essere minacciati se non dall'umanità stessa. Luogo e soggetto di un' ambivalenza irrisolta, l'umanità si presenta come portatrice di una sua minaccia ma anche della sua neutralizzazione; lavora per la pace" (RESTA, Eligio. L'inimicizia, l'umanità, le guerre. Sociologia del diritto, n. 1, 2001. p. 12-13).

16 "Immigration has grown a lot in the last 20 years and a significant fraction of this growth has been from other EU countries, especially after 2004 and the accession of eight East Europeancountries (the 'A8'). Between 1995 and 2015, the number of immigrants from other EU countries living in the UK tripled from 
Un altro fattore, non meno rilevante, che occorre mettere in risalto è il ruolo dei mezzi di comunicazione, che hanno concorso nel consolidare la certezza che avvenisse esattamente il contrario di ciò che è poi realmente accaduto, tanto riguardo alla Brexit quanto relativamente ad altri eventi di significativo impatto globale. Questa constatazione ci porterebbe ad interrogarci sulla democrazia e sulle procedure di voto: per riflettere su come si vota, e a chi vota per chi? o per cosa? Basti pensare al cd. urban-rural divide, al peso che per il voto sulla Brexit hanno avuto le "periferie". Fino a tempi recenti non si dava molto peso a queste differenze, che in effetti si sono rilevate pesantissime (si pensi anche al voto per le elezioni presidenziali americane ed all'elezione di Donald Trump). Oggi si sono invece rivelate fondamentali, soprattutto nel consolidare l'impressione che ci si orienti al voto più in base alle aspettative che in base al contenuto delle scelte.

La sfera pubblica tende in effetti a basarsi sulle aspettative delle aspettative, piuttosto che non sui contenuti: ed il voto nel Regno Unito ha rivelato spunti critici in tal senso, anche laddove si è potuto rilevare che che se si fosse votato in un giorno differente da quello prescelto, il risultato dle voto avrebbe potuto essere diverso. Oltre al caso di Donald Trump, già richiamato, anche il voto per le elezioni presidenziali in Brasile è stato fortemente orientato da coloro che ritenevano di poterne trarre qualche beneficio. Dilma Roussef deve, infatti, la sua elezione al significativo spostamento delle intenzioni di voto a causa dell'interesse suscitato dagli scandali per la corruzione, dalla aspettativa per un preteso cambiamento rispetto al passato. Con la stortia che ha poi dimostrato, per quanto all'improvviso, esattamente il contrario.

Tanto in America Latina, così come in altri Paesi periferici, cosa ci si può aspettare da un referendum? L'espressione della effettiva “volontà popolare"? Si potrebbe rispondere, brutalmente di no. Sempre il peggio! Come già sottolineato da Weber e da Luhmann, i procedimenti di voto pesano molto di più del consenso popolare. E' sempre più evidente che, da un lato, i partiti scompaiono, o perdono la loro capacità di mediazione e di sintesi, e sempre di più acquistano importanza i procedimenti decisionali, i sistemi elettorali, i processi referendari. La stessa crisi democratica evidentemente in atto nei Paesi dell'Unione europea a causa della scomparsa dei partiti tradizionali (basti pensare all'Italia, alla Spagna ed alla Grecia) che trascina la distruzione dell'organizzazione della vita pubblica e dei

0.9 million to 3.3 million. The share of EU nationals grew from $1.5 \%$ to $5.3 \%$ of the total population and from $1.8 \%$ to $6.3 \%$ of the working age population (adults aged 16-64)". Questi dati sono tratti dall'articolo WADSWORTH, Jonathan et al. Brexit and the Impact of Immigration on the UK. London: Centre for Economic Performance; London School of Economics and Political Science. Disponível em: <http://cep.Ise.ac.uk/ pubs/download/brexit05.pdf>. Acesso em: 27 jun. 2017. 
processi selettivi delle finalità politiche, il cedimento del diritto amministrativo, e così via, ha ormai raggiunto i Paesi della periferia della modernità.

L'Unione europea sta in effetti scontando il deteriorata mento della propria immagine ed il Regno Unito ha maturato una reazione scomposta, mal orientata e poco meditata il cui effetto è stato un voto basato appunto su aspettative di miglioramento della qualità della vita, piuttosto che sui contenuti reali della decisione aassunta per via referendaria. Nei Paesi del Mercosul è possibile osservare una stessa situazione di stallo, con celebrazione di votazioni anch'esse orientate dalle aspettative e non dai reali contenuti, espressi nei programmi e plausibili secondo realtà.

Come affermato da von Jhering, ${ }^{17}$ la società è un'organizzazione effettiva della vita per e attraverso gli altri. Nella misura in cui la vita nella società è possibile soltanto attraverso il riconoscimento dell'altro, occorre identificare le nuove implicazioni sociali degli sviluppi descritti. In questa prospettiva analitica in questo lavoro ci si limita agli esiti del referendum sulla Brexit, provando a valutarne i possibili effetti sul Mercosul in una prospettiva non esclusivamente giuspolitica.

\section{II referendum sulla Brexit e i suoi riflessi sul Mercosul}

L'imprevisto risultato del referendum celebratosi il 23 giugno 2016 nel Regno Unito ha generato una crisi costituzionale ${ }^{18}$ che i più attenti tra gli studiosi non

17 Si veda, JHERING, R. von.; PIOVANI, P. (A cura di). La lotta per il diritto. Bari: Laterza, 1960

18 Nei Paesi dell'America Latina, le crisi costituzionali sono un fenomeno costante, E le riforme un processo permanente. Nel caso del Brasile, la Costituzione del 1988 è già stata emendata più di novanta volte, più precisamente 96, l'ultima delle quali in relazione all'Art. 225, riportato di seguito. "Todos têm direito ao meio ambiente ecologicamente equilibrado, bem de uso comum do povo e essencial à sadia qualidade de vida, impondo-se ao Poder Público e à coletividade o dever de defendê-lo e preservá-lo para as presentes e futuras gerações.

$\S 1^{\circ}$ Per garantire l'effettività di questo diritto, è compito del Governo:

I. preservare e restaurare i processi ecologici essenziali e provvedere alla gestione ecologica delle specie e ecosistemi;

II. preservare la diversità e l'integrazione del patrimonio genetico del Paese e controllare gli enti dedicati alla ricerca e manipolazione di materiale genetico;

III. definire, per tutte le unità della Federazione, spazi territoriali e loro componenti che necessitano di particolare protezione, essendone consentita l'alterazione e soppressione solo attraverso la legge, con il divieto di qualsiasi utilizzo che comprometta l'integrità delle caratteristiche che giustificano la protezione; IV. esigere, secondo quanto previsto dalla legge, per l'installazione di opere o attività potenzialmente portatrici di significativo degrado ambientale, uno studio preventivo di impatto ambientale, a cui verrà dato ampio risalto;

V. controllare la produzione, la commercializzazione e l'impiego di tecniche, metodi e sostanze che comportano rischi per la vita, la qualità di vita e l'ambiente;

VI. promuovere l'educazione ambientale in tutti i livelli di insegnamento e formare una coscienza pubblica per la preservazione dell'ambiente; 
hanno esitato a definire la più grave crisi del sistema politico-istituzionale inglese a far data dal $1688 .{ }^{19}$ Un episodio, quello del giugno 2016, che ha in effetti messo in discussione lo stesso cardine del sistema costituzionale britannico, quel principio della Sovereignty of Parliament che ne rappresenta il principale fondamento. ${ }^{20}$ Una vicenda che mette in discussione funditus la stessa nozione di democrazia rappresentativa, generando una $\mathrm{crisi}^{21}$ del suo stesso principio di legittimazione al cospetto della sempre attuale degenerazione populista della stessa democrazia, ${ }^{22}$ in una con i fondamenti del processo di integrazione europea e i fondamentali assetti attuali del regime dell'UE. Anche comparando questa vicenda con “crisi” differenti, se fino ad ora può sostenersi che in generale I'UE sia riuscita a superare

VII. proteggere la fauna e la flora, con il divieto, nella forma di legge, di compiere azioni che mettano in pericolo la loro funzione ecologica, provochino l'estinzione di specie o sottopongano gli animali a crudeltà. $\S 20$ Chi sfrutta risorse minerali ha l'obbligo di risanare l'ambiente degradato, in accordo con la soluzione tecnica richiesta dall'organismo pubblico competente, secondo quanto stabilito dalla legge.

§3ㅇ I comportamenti e le attività considerate lesive per l'ambiente assoggetteranno i loro autori, persone fisiche o giuridiche, a sanzioni penali e amministrative, indipendentemente dall'obbligo di riparare i danni causati.

§4느 La Foresta Amazzonica brasiliana, la Foresta Atlantica, la Serra del Mar, il Pantanal Mato-Grossense e la Zona Costiera sono patrimonio nazionale, ed il loro utilizzo avverrà, secondo quanto stabilito dalla legge, nell'ambito di condizioni che garantiscano la preservazione dell'ambiente, soprattutto per quello che riguarda l'uso delle risorse naturali.

$\S 5$ 은 Sono indisponibili le terre disabitate o sotto la custodia degli Stati sottoposti a processi discriminanti, necessari alla protezione degli ecosistemi naturali.

§6 La localizzazione delle fabbriche che operano con reattori nucleari dovrà essere definita per legge federale, senza la quale non potranno essere installate".

19 Così D. Galligan, The Constitution in Crisis 2016, nella Conferenza tenuta lo scorso 8 dicembre 2016 presso il Wolfson College, Oxford, la cui sintesi è disponibile sul sito web de The Foundation for Law, Justice and Society, all'indirizzo <http://www.fljs.org/content/professor-denis-galligan-invokes-spiritputney-debates-confront-constitutional-challenges $>$, ove è disponibile anche il podcast con la registrazione della conferenza, <http://www.fljs.org/content/constitution-crisis-2016>.

20 Rinvio, alle considerazioni svolte ed alla importante letteratura diffusamente citata in Sovranità, Relazione al XXXI Convegno annuale dell'Associazione italiana dei costituzionalisti (AIC) sul tema Di alcune grandi categorie del Diritto costituzionale: Sovranità, Rappresentanza, Territorio, Trento 11-12 novembre 2016 (BILANCIA, Francesco. Sovranità. Rivista AIC, n. 3, 2017).

21 Una crisi che oltrepassa l'Unione europea e colpisce direttamente i Paesi del Mercosul: la Brexit instilla dubbi anche nell'atteso accordo di libero scambio fra Mercosul ed Unione Europea. II Presidente brasiliano in carica, Michel Temer, venerdì 24 giugno 2016 ha detto di considerare il Mercosul "importante" ma che, nella visione del governo, occorre ridiscutere i contenuti dell'accordo per realizzare l'obiettivo della politica esterna del governo di ampliare le relazioni con il maggior numero possibile di Paesi nel mondo. Ci sono cinque possibili effetti economici dell'uscita del Regno Unito dalla UE. Gli esperti sottolineano che il rischio principale è rappresentato dall'uscita di altri Stati-membri. L'indebolimento dell'UE potrebbe inoltre avere importanti conseguenze per altri Paesi.

22 Cosi, BRUNELLI, G. et al. Dal governo democratico-rappresentativo al governo a mera legittimazione popolare. (Brevi riflessioni sui mutamenti di regime). In: BRUNELLI, Giuditta; PUGIOTTO, Andrea; VERONESI, Paolo (A cura di). Scritti in onore di Lorenza Carlassare. Napoli: Jovene, 2009. p. 1801 ss.; nonché in BILANCIA, Francesco et al. Le forme della democrazia contemporanea e il germe della sua autodistruzione. In: GIANELLI, A. et al. (A cura di). Democrazia. Storia e crisi di una forma politica. Napoli: Editoriale Scientifica, 2013. p. 135 ss. 
molti importanti problemi emersi nel corso del suo lungo processo di integrazione, finendo col risolvere in modo relativamente soddisfacente le proprie questioni interne, la "sorpresa” dell'uscita del Regno Unito ha scosso in maniera significativa la struttura politica, giuridica, sociale e economica del sistema stesso, implicando direttamente questioni sociali e provocando un impatto negativo su altri "mercati" e sistemi istituzionali regionali, come ad esempio il Mercosul. In un serrato confronto tra sistemi, in effetti, il modello di integrazione europea non ha contribuito in alcun modo alla affermazione di un modello generale di integrazione. Non che si fosse inconsapevoli del complesso rapporto del Regno Unito con I'UE stessa; tuttavia nemmeno chi ha proposto il referendum si aspettava questo risultato. II Premier Cameron, proponendo la consultazione referendaria, non aveva neanche considerato la eventualità di una vittoria del "no"; ha dimostrato di non aspettarsi che si potesse verificare quel che è accaduto. E' vero che già in passato il Regno Unito avesse più volte chiesto a Bruxelles di porre limiti ai flussi migratori intracomunitari e che i cittadini del Regno Unito avessero frequentemente reclamato una maggiore autonomia dall'UE. Ma ciò non è stato sufficiente a rendere in effetti prevedibile l'esito del voto.

Per l'Europa e per il mondo, ${ }^{23}$ anche a seguito dell'elezione di Donald Trump come quarantacinquesimo Presidente degli Stati Uniti, si apre in effetti una stagione storica di grandi incertezze, ${ }^{24}$ nel corso della quale iniziano già a manifestarsi i primi gravi sintomi di un ritorno al nazionalismo, al protezionismo statale ed ai populismi forieri di pesanti minacce nei confronti dei processi di globalizzazione dei mercati e di composizione di un'Unione europea coesa e solidale. In proposito non resterebbe che attendere gli sviluppi delle politiche ispirate da tali nuovi "valori", al fine di valutarne gli esiti sugli assetti istituzionali e costituzionali delle principali organizzazioni internazionali, ${ }^{25}$ in primo luogo ONU e WTO, ovviamente della stessa Unione europea e degli Stati costituzionali contemporanei, come nel caso dei Paesi del Mercosul, che vedono nel Trattato istitutivo dell'Unione europea un modello per

23 Eventi che si verificano in un punto qualsiasi del mondo hanno influenza su tutto il pianeta. Si noti che il referendum UK ha avuto effetti non solo nell'Unione europea ma anche nelle economie dei Paesi emergenti, dove l'impatto sulle borse valori è stato molto più forte.

24 La società contemporanea è caratterizzata dal rischio, e dalla necessità di prendere decisioni rilevanti. Si potrebbe sempre decidere diversamente rispetto a quel che realmente si fa, e l'impatto di ciò sul sistema e sulle poliche pubbliche è enorme. II rischio, come paradosso costitutivo della modernità della società contemporanea, viene presentato come un dato, un'informazione oggettiva, vincolata a decisioni e azioni future. Cfr. su questo LUHMANN, Niklas. Sociologia del rischio. Milano: Bruno Mondadori, 1996.

25 Su questo tema si veda ZOLO, Danilo. Globalizzazione. Una mappa dei problemi. Bari: Laterza, 2009. p. 88. “Ai processi di globalizzazione si accompagna una graduale trasformazione non solo delle strutture della politica ma anche degli apparati normativi, anzitutto del diritto internazionale. [...] Accanto agli Stati e alle tradizionali istituizioni sovranazionali, come le Nazioni Unite, il Fondo Monetario Internazionale, la Banca Mondiale, l'Organizzazione Mondiale del Commercio, si profilano nuovi soggetti dell'ordinamento giuridico internazionale". 
avvicinare i cittadini ai processi decisionali, nonché una proposta per "organizzare relazioni tra Stati in modo coerente e solidaristico". Altre regioni del globo si sono sempre lasciate ispirare dalle espressioni contenute nei documenti dell'UE, specialmente dalle idee e dai principi di solidarietà, cooperazione, coerenza, sviluppo sostenibile, qualità della vita.

Una premessa che serve ad inquadrare un clima politico, un'atmosfera composta dal precipitato del discorso pubblico che sembra caratterizzare il tempo presente nel lasciar ispirare gli sviluppi del sistema di relazioni istituzionali all'interno ed all'esterno degli ordinamenti statali, nell'Unione europea e nei principali contesti istituzionali sovranazionali. Per poter dire, in estrema sintesi, che questi sono oggi i temi sul tappeto, ${ }^{26}$ i principali argomenti del discorso politico e degli sviluppi istituzionali delle conseguenti politiche pubbliche e che è pertanto con queste tematiche che anche le scienze sociali devono oggi misurarsi.

La Brexit rappresenta un evento chiave per la storia della integrazione/ disintegrazione: è la prima esperienza di questo tipo, e sebbene nell'art. 50 del Trattato di Lisbona fosse prevista questa possibilità, non ci si aspettava che si verificasse; almeno fino ad oggi, osservando del resto le difficoltà nella gestione di tale crisi, certamanente una delle più gravi vissute fino ad oggi dai "sistemi istituzionali regionali". Tale situazione ha come conseguenza, tra le altre, anche la riduzione dell'accesso ai diritti, in particolare ai diritti sociali.

Al contrario di quanto previsto nell'Unione europea, i trattati istitutivi e i protocolli del Mercosul ${ }^{27}$ non fanno riferimento a referendum per l'uscita di uno dei Paesi dall'accordo. Tuttavia nel 2012 Carta Capital ha dato notizia del fatto che il Paraguay avesse preso in considerazione l'ipotesi di un referendum per decidere se rimanere nell'accordo oppure no, quale conseguenza della misura della sospensione subita tanto in riferimento al Mercosul quanto alla UNASUL (I'Unione delle Nazioni Sudamericane). Tuttavia la rivista non ha specificato su quali basi il governo del Paraguay avesse sollevato l'ipotesi né se tale consultazione si fosse effettivamente svolta. ${ }^{28}$

26 I temi che vengono nascosti sotto il tappeto nella modernità necessitano di essere disvelati, per presentarsi poi nella forma di paradossi.

27 II Mercosul rappresenta un processo di integrazione iniziato nel 1991 con la firma del Tratado de Assunção da parte di Argentina, Brasile, Paraguay e Uruguay. Con l'instituzione del Protocollo di Ouro Preto, nel 1994, il blocco economico ha acquisito personalità giuridica internazionale. Nel 2012 il Venezuela è divenuto Stato membro. Anche nel 2013, fino alla metà dell'anno, il Paraguay ha subito una sospensione a causa del mancato rispetto della clausola democratica. Del Mercosul fanno parte anche la Bolivia, che è in fase di adesione fin dal 7 dicembre 2012 e, come Stati associati, Cile, Perù, Colombia, Ecuador, Guiana e Suriname. Sulla storia del Mercosul, cfr.: SAIBA mais sobre o Mercosul. Mercosul. Disponível em: <http:// www.mercosul.gov.br/saiba-mais-sobre-o-mercosul\#DADOSGERAIS>. Acesso em: 27 jun. 2017.

28 Su questo, GIRALDI, Renata. Paraguai cogita referendo para definir permanência no Mercosul. Carta Capital, São Paulo, 15 ago. 2012. Disponível em: <https://www.cartacapital.com.br/internacional/ paraguai-cogita-referendo-para-definir-permanencia-no-mercosul>. Acesso em: 28 jun. 2017. In questo 
Questo precedente può, in effetti, indurre a ritenere che anche nel Mercosul la secessione di uno degli Stati membri non sia giuridicamente proibita, ancorché neanche espressamente consentita. ${ }^{29}$ II Mercosul ha previsto solamente la possibilità della sospensione di uno Stato-membro, per iniziativa degli altri, quando sia stata accertata una violazione della clausula democratica, secondo il "Protocolo de Montevideu Sobre o Compromisso com a Democracia no Mercosul" ["Protocollo di Montevideo sull'impegno per la democrazia nel Mercosul”]. ${ }^{30}$

senso è importante fare riferimento alla notizia diffusa, sempre da Carta Capital, nell'agosto del 2016, "A terra treme no Mercosul" ("La terra trema nel Mercosul”), in cui si fa menzione del rischio di una presidenza vacante una volta terminato il periodo di occuppazione della stessa da parte dell'Uruguay, in un momento di intensa polemica sul Venezuela: una situazione descritta come il periodo di crisi più profonda nella storia dell'accordo, dalla sua creazione nel 1991 (AMORIM, Celso. A terra treme no Mercosul. Carta Capital, São Paulo, 1ํago. 2016. Disponível em: <www.cartacapital.com.br/politica/a-terra-treme-nomercosul>. Acesso em: 27 jun. 2017).

29 BADR, Eid. O direito comunitário e o Mercosul à luz da Constituição Federal brasileira. Curitiba: CRV, 2011.

30 Secondo l'articolo 6으 del Protocollo: “Em caso de ruptura ou ameaça de ruptura da ordem democrática em uma Parte do presente Protocolo, os Presidentes das demais Partes ou, na falta destes, seus Ministros das Relações Exteriores em sessão ampliada do Conselho do Mercado Comum poderão estabelecer, dentre outras, as medidas que se detalham a seguir: a.- Suspender o direito de participar nos diferentes cargaos da estrutura institucional do Mercosul. b.- Fechar de forma total ou parcial as fronteiras terrestres. Suspender ou limitar o comercio, o trafego aéreo e marítima, as comunicações e o fornecimento de energia, serviços e abastecimento. c.- Suspender a Parte afetada do gozo dos direitos e benefícios emergentes do Tratado de Assunção e seus Protocolos e dos Acordos de integração celebrados entre as Partes, conforme couber. d.- Promover a suspensão da Parte afetada no âmbito de outras organizações regionais e internacionais. Promover junto a terceiros países ou grupos de países a suspensão da Parte afetada de direitos e/ou benefícios derivados dos acordos de cooperação dos quais seja parte. e.- Respaldar os esforços regionais e internacionais, em particular no âmbito das Nações Unidas, encaminhados a resolver e a encontrar uma solução pacifica e democrática para a situaçao ocorrida na Parte afetada. f.- Adotar sançoes políticas e diplomáticas adicionais. As medidas guardarão a devida proporcionalidade com a gravidade da situação existente; não deverão per em risco o bem-estar da população e o gozo efetivo dos direitos humanos e liberdades fundamentais ria Parte afetada; respeitarão a soberania e integridade territorial da Parte afetada, a situação dos países sem literal marítimo e os tratados vigentes" ("In caso di rottura o di minaccia di rottura dell'ordine democratico in uno degli Stati parte del presente Protocollo, i Presidenti degli altri Stati o, in loro assenza, i loro Ministri degli Esteri in sessione allargata del Consiglio del Mercato Comune possono stabilire, di comune accordo, le misure di seguito dettagliate: a.- La sospensione del diritto a partecipare nei differenti ambiti della struttura istituzionale del Mercosul. b.- La chiusura totale o parziale delle frontiere terrestri. La sospensione o la limitazione del commercio, del traffico aereo e marittimo, delle comunicazioni e della distribuzione dell'energia, dei servizi e dei rifornimenti. c.- La sospensione, per la parte interessata, del godimento dei diritti e dei benefici conseguenti al Tratado de Assunção e di protocolli ed accordi di integrazione siglati tra le parti, a seconda dei casi. D.- La sospensione della Parte dalle altre organizzazioni regionali e internazionali. La promozione, unitamente a Paesi terzi o a gruppi di Paesi, della Parte dai diritti e/o dai benefici derivanti da accordi di cooperazione di cui è parte. E.- Il sostegno di sforzi regionali e internazionali, in particolare in seno alle Nazioni Unite, tesi a risolvere ed a trovare una soluzione pacifica e democratica della situazione verificatasi nella Parte interessata. F.- L'adozione di sanzioni politiche e diplomatiche aggiuntive. Le misure dovranno avere la giusta proporzionalità in rapporto alla gravità della situazione; non dovranno mettere a rischio il benessere della popolazione e l'effettivo godimento dei diritti umani e delle libertà fondamentali della Parte interessata; rispetteranno la sovranità e l'integrità territoriale della Parte, la condizione dei Paesi privi di accesso al mare ed i trattati vigenti") (PROTOCOLO de Montevideu Sobre o Compromisso com a Democracia no Mercosul. Mercosul. Disponivel em: <dai-mre.serpro.gov.br/atos-internacionais/ multilaterais/protocolo-de-montevideu-sobre-compromisso-com-a-democracia-no-mercosul-ushuaia-iifirmado-por-ocasiao-da-xlii-reuniao-do-cmc-e-cupula-de-presidentes-do-mercosul-e-estados-associados $/>$. Acesso em: 27 jun. 2017). 
Nel Mercosul l'obbligo del rispetto dell'ordine democratico a livello statale è stato incluso nel trattato alla luce della lunga storia di sconvolgimenti e colpi di Stato militari nei Paesi del blocco economico. ${ }^{31}$ Questa importante decisione è riuscita a vincolare ai valori della democrazia il processo di integrazione, ed è stata invocata nel caso del Paraguay nel 2012-2013 e per il Venezuela nel 2016, ${ }^{32}$ anche come strumento al fine di garantire i diritti sociali dei cittadini degli Stati membri.

Il taglio della presente riflessione ha preso, dunque, le mosse proprio dal discorso politico e dai principali argomenti della campagna referendaria sull'uscita del Regno Unito dall'Unione europea; come pure dal tenore delle questioni di fondo che caratterizzano il dibattito contemporaneo sulla crisi di legittimazione dell'Unione europea - ${ }^{33}$ non solo nel Regno Unito - e che presumibilmente orienteranno anche il dibattito e le future scelte in merito alle modalità di attuazione di questa così grave ed importante decisione degli elettori britannici. Malgrado l'evidente, fondamentale, importanza assunta da altri temi e questioni emergenti dalla crisi innescata dalla scelta del Regno Unito di attivare la procedura di cui all'art. 50 del Trattato sull'Unione europea per recedere dai trattati, la attenzione è qui volta prevalentemente alla richiamata questione del rapporto tra libertà di circolazione dei cittadini dell'UE e garanzia dei diritti sociali nel sistema del mercato interno. Non prenderemo pertanto in considerazione né la interessantissima questione, piena di fondamentali implicazioni per il sistema costituzionale del Regno Unito, del conflitto in atto tra principio della sovranità parlamentare e prerogativa regia, nel frattempo risolto dalla decisione della Corte suprema del Regno Unito con

31 Su questo cfr.: LORENZETTI, Ricardo. Sistema Jurídico del Mercosur. In: ARAUJO, Nadia de; MARQUES, Claudia Lima (Org.). O novo direito internacional: estudos em homenagem a Erik Jayme. Rio de Janeiro: Renovar, 2005. p. 652. "[...] la democracia como forma de gobierno surge como un principio estrutural, de modo que su violacion resulta impeditiva y aun excluyente de um Estado-parte que suprima esta forma de gobierno. Es una importante decisión del legislador mercosuriano, que frente a uma historia de convlusiones y golpes militares en la zona, há resuelto vincular la integracion com la decomcracia, como uma manera de desalentar el regresso al passado" "Ila democrazia come forma di governo si presenta quale principio strutturale, cosicché la sua violazione rappresenterebbe un impedimento ed una causa escludente per gli Stati membri che sopprimessero tale forma di governo. Si tratta di una decisione importante nell'elaborazione del trattato del Mercosul, che di fronte ad una storia fatta di violenze e golpe militari, ha deciso di vincolare l'integrazione alla democrazia, in maniera da scoraggiare il ritorno al passato").

32 MERCOSUL suspende Paraguai e anuncia adesão da Venezuela. G1, 29 jun. 2012. Disponível em: <http://g1.globo.com/mundo/noticia/2012/06/mercosul-suspende-paraguai-e-anuncia-adesao-davenezuela.html>. Acesso em: 27 jun. 2017; BRASIL, Argentina, Paraguai e Uruguai suspendem Venezuela do Mercosul. Revista Valor Econômico, 1ํjan. 2016. Disponível em: <http://www.valor.com.br/ brasil/4793901/brasil-argentina-paraguai-e-uruguai-suspendem-venezuela-do-mercosul>. Acesso em: 27 jun. 2017.

33 Esemplare sembra, in effetti, la riflessione aperta da HARLOW, Carol. The limping legitimacy of EU Lawmarking: a barrier to integration. Eur Papers, 2016. p. 29 ss. 
la nota sentenza sul caso Miller. ${ }^{34}$ Con l'affermazione cioè della necessità che fosse un intervento legislativo del Parlamento a definire i contorni del mandato del governo a negoziare con gli altri partner europei - ed in seno alle istituzioni UE - le modalità ed i passaggi per la formazione e la definizione dei contenuti del trattato di cui all'art. 50, secondo comma del TUE. ${ }^{35}$ Né prenderemo in considerazione la questione dei termini temporali per la definizione dei contenuti di tale decisione e la sua notifica alle istituzioni dell'UE. Così come non svilupperemo una riflessione, a monte e con effetti condizionanti rilevantissimi sulla questione in corso, sul significato del referendum in una prospettiva sociologico-giuridica, né tanto meno con riguardo agli effetti di tale tipo di atto giuridico sul sistema politico e la forma di governo del Regno Unito. ${ }^{36}$ Sarebbe infatti molto interessante interrogarsi, ad esempio, sulle cause e le implicazioni della decisione del corpo elettorale in una prospettiva socio-legal, con attenzione alle forti diseguaglianze sul piano sociale, culturale e politico in seno alla società del Regno Unito che indubbiamente sono sembrate emergere dai risultati del referendum in relazione alla distribuzione territoriale dei voti a favore o contro la proposta, indicando significative ma

34 UKSC (5) 2017 del 24 gennaio 2017. The Supreme Court, 2017. Disponível em: <https://www. supremecourt.uk/cases/docs/uksc-2016-0196-judgment.pdf>. Acesso em: 27 jul. 2017, su cui almeno KING, J. What next? Legislative authority for triggering art. 50. UK Constitutional Law Association. Disponível em: <https://ukconstitutionallaw.org/2017/01/24/jeff-king-what-next-legislative-authority-for-triggeringarticle-50-2/>. Acesso em: 27 jun. 2017; FABBRINI, F. Brexit according to the UK Supreme Court: the Miller Judgement. Centro Studi Sul Federalismo, n. 100, 27 gennaio 2017; BRADLEY, A. Pressure on a Historical Constitution: the Brexit decision in the UK's Supreme Court. Dir.pubbl., n. 1, 2017. p. 3 ss.; LEYLAND, P. Brexit and the UK: charting the constitutional and legal obstacles. Istituzioni del Federalismo, 2017. In corso di pubblicazione; SGRÒ, F. II caso "Brexit": qualche considerazione sulla sovranità parlamentare e sul sistema delle fonti nell'ordinamento costituzionale britannico dopo la sentenza della Supreme Court of the United Kingdom. Federalismi.it, n. 5, 2017. Per il seguito legislativo della sentenza, concluso in data 17 marzo 2017 con la pubblicazione del EUROPEAN Union (Notification of Withdrawal) Act 2017. Legislation.gov.uk. Disponível em: <http://www.legislation.gov.uk/ukpga/2017/9/enacted>. Acesso em: 27 jun. 2017, rinviamo alla utile ed aggiornata rassegna di cui all' OSSERVATORIO sulla Brexit 25 maggio 21 giugno 2017. Federalismi.it. Disponivel em: <http://www.federalismi.it/nv14/articolo-documento. cfm?Artid=32932>. Acesso em: 27 jul. 2017.

35 Una rassegna delle questioni aperte è, ad esempio, rinvenibile nel rapporto elaborato dalla House of Commons, Exiting the European Union Committee, The Process for exiting the European Union and the Government's negotiating objectives, HC 815, 14 gennaio 2017, nonché con toni in parte propagandistici, nel Rapporto del Governo dal titolo The United Kingdom's Exit from and new Partnership with the European Union, presented to Parliament by the Prime Minister by Command of Her Majesty nel febbraio 2017 (THE United Kingdom's exit from and new partnership with the European Union. Gov.uk, febbraio 2017. Disponível em: <https://www.gov.uk/government/publications/the-united-kingdoms-exit-from-and-newpartnership-with-the-european-union-white-paper>. Acesso em: 27 jul. 2017).

36 Sul modello della riflessione in Italia magistralmente condotta da MEZZANOTTE, C.; NANIA, R. Referendum e forma di governo in Italia. Dem.dir., 1981. p. 51 ss. e da LUCIANI, M. Art. 75. II referendum abrogativo. In: BRANCA, Giuseppe (A cura di). Commentario della Costituzione. Bologna: N. Zanichelli; Roma: Soc. Ed. del Foro Italiano, 2005, spec. p. 1 ss., p. 122 ss. nonché, sul valore del referendum nel sistema degli atti normativi, p. 599 ss. 
territorialmente omogenee diseguaglianze. ${ }^{37}$ Ancora, per lo studioso di diritto costituzionale $^{38}$ questa vicenda solleciterebbe una riflessione sui delicati equilibri tra democrazia diretta e democrazia rappresentativa, sui rapporti sempre più conflittuali tra popolo-corpo elettorale ed istituzioni (starei per dire componenti) del Parlamento, e così via.

Ma in questa sede nessuna di queste importanti prospettive è oggetto di analisi, così come non si assumerà a riferimento della riflessione la connessa "questione economica", declinabile per un verso in riferimento ai possibili futuri assetti del mercato comune, e per altro con più specifico riguardo alle conseguenze del processo di disgregazione in atto sui mercati finanziari con i probabili connessi esiti in termini di squilibri monetari tra le diverse aree del mercato stesso, ${ }^{39}$ che oltrepassa le frontiere della UE e ha riflesso anche in altre regioni e su altri mercati integrati in accordi regionali, come il Mercosul.

Basti pensare ai possibili effetti della Brexit sugli accordi di libero scambio conclusi dall'Unione europea, come nel caso del Mercosul, per quanto ancora in fase di negoziazione. ${ }^{40}$ La tabella seguente mostra il potenziale impatto economico di questo fenomeno proprio nei confronti del Mercosul, per quanto in termini percentuali al cospetto dell'Unione europea questo assumerebbe dimensioni

37 Rinviamo agli interessantissimi spunti di analisi di cui all'intervento sulla questione di Jürgen Habermas, CORE Europe to the rescue: a conversation with Jürgen Habermas about Brexit and the EU crisis. Social Europe, 12 jul. 2016. Disponivel em: <https://www.socialeurope.eu/core-europe-to-the-rescue>. Acesso em: 27 jun. 2017, ad esempio a proposito del conflitto tra populismo e capitalismo, o del c.d. urban-rural divide.

38 Sociologia das constituições.

39 Cfr. su questo Impact of Brexit on Europe-China Relations, published 10 October, 2016. "Foreign direct investment (FDI) from China into the EU as whole reached US\$23 billion in 2015 even as the EU's outward flows declined, with new investments up $28 \%$ compared with the previous year's figures. Traditionally, this has been largely market-seeking FDI as Chinese firms have sought overseas bases from which to pursue ambitious global expansion strategies, with the EU being seen as a key opportunity. However, there is a growing trend in technology-seeking FDI through mergers, acquisitions and joint collaboration between European firms and Chinese partners keen to tap into the EU's high levels of innovation capability" ("Gli investimenti diretti esteri (FDI) dalla Cina nell'UE nel complesso hanno raggiunto i 23 miliardi di dollari nel 2015, mentre i flussi esterni dell'UE sono diminuiti, con nuovi investimenti fino al $28 \%$ maggiori rispetto ai dati dell'anno precedente. Tradizionalmente, ciò è dovuto in gran parte alla ricerca di mercati orientati dai FDI in quanto le imprese cinesi hanno cercato basi oltremare da cui perseguire ambiziose strategie di espansione globale, con l'UE considerata un'occasione fondamentale. Tuttavia, vi è una tendenza crescente nel campo delle tecnologie riguardanti gli FDI attraverso fusioni, acquisizioni e collaborazioni congiunte tra imprese europee e partner cinesi che vogliono sfruttare gli alti livelli di capacità di innovazione dell'UE").

40 Notizie recenti indicano un'evoluzione dei negoziati sull'accordo Mercosul-UE. Cfr.: OLIVEIRA, R.; MARREIRO, F. UE e Mercosul correm para fechar acordo antes do avanço dos nacionalismos. Jornal El País, 6 maio 2017. Disponível em: <https://brasil.elpais.com/brasil/2017/05/05/internacional/1494017908_326426. html>. Acesso em: 10 jul. 2017; HÁ 'otimismo realista' com acordo entre Mercosul e UE, diz secretário. Revista Época Negócios, 6 jul. 2017. Disponível em: <http://epocanegocios.globo.com/Mundo/ noticia/2017/07/ha-otimismo-realista-com-acordo-entre-mercosul-e-ue-diz-secretario.html>. Acesso em: 10 jul. 2017; NEGOCIADOR quer fechar ponto chave de acordo UE-Mercosul esse ano. Revista Exame, 6 jul. 2017. Disponível em: <http://exame.abril.com.br/economia/negociador-quer-fechar-ponto-chave-deacordo-ue-mercosul-esse-ano/>. Acesso em: 10 jul. 2017. 
sproporzionatamente piccole, non solo perché l'economia dell'UE è di dimensioni molto maggiori di quella del sistema del Mercosul, ma anche perché le aziende che in essa hanno sede detengono un maggiore potere di mercato.

Secondo Ansgar Belke e Daniel Gros, tutti gli studi attualmente disponibili mostrano rischi di riduzione/interruzione delle relazioni commerciali e l'imporsi di costi per entrambe le parti, anche se per l'Unione europea tali costi siano minori. ${ }^{41}$ Ragione per cui i risultati del referendum e delle successive elezioni generali nel Regno Unito, insieme alla grande incertezza derivante da esse, segnalano una tendenza all'incremento dei costi economici derivanti dalla Brexit per lo stesso Regno Unito, costi senz' altro maggiori rispetto alle potenziali perdite specularmente gravanti sull'Unione Europea. Si veda la seguente tabella:

\section{Tabella: Simulazione dell'impatto economico della BREXIT sull'accordo UE-Mercosul ${ }^{42}$}

\begin{tabular}{|c|c|c|c|}
\hline & Scenari possibili & $\begin{array}{r}\text { Effetti di lungo ra } \\
\text { assoluti e per }\end{array}$ & $\begin{array}{l}\text { şio nel PIB: } \\
\text { entuale }\end{array}$ \\
\hline $\begin{array}{l}\text { EU- } \\
\text { Mercosul }^{43}\end{array}$ & $\begin{array}{l}\text { Simulazione dei } \\
\text { risultati } \\
\text { Modello di equilibrio } \\
\text { economico generale } \\
\text { computazionale } \\
(\mathrm{CGE})^{44} \\
\text { Scenario di riferi- } \\
\text { mento = proposta } \\
\text { Dell'Unione europea }\end{array}$ & $\begin{array}{l}\text { EU12: }+273.86 \text { mld } € \\
\text { nei prezzi del } 2020 \\
\text { EU15: }+5,214.39 \text { mld } \\
€ \text { nei prezzi del } 2020 \\
\text { MERCOSUR: } \\
+1,523.28 \text { mld } € \text { nei } \\
\text { prezzi del } 2020 \\
=\text { Differenza assoluta } \\
\text { nello scenario di riferi- } \\
\text { mento }\end{array}$ & $\begin{array}{l}\text { EU12: +0.02 \% } \\
\text { EU15: +0.02 \% } \\
\text { MERC0SUR: } \\
+0.16 \% \\
=\text { Percentuale } \\
\text { di differenza } \\
\text { nello scenario } \\
\text { di riferimento }\end{array}$ \\
\hline
\end{tabular}

41 BELKE, Ansgar; GROS, Daniel. The economic impact of Brexit: evidence from modelling free trade agreements. Brussels, 2017. Disponível em: <http://www.rwi-essen.de/media/content/pages/ publikationen/ruhr-economic-papers/rep_17_700.pdf>. Acesso em: 10 jul. 2017.

42 BELKE, Ansgar; GROS, Daniel. The economic impact of Brexit: evidence from modelling free trade agreements. Brussels, 2017. Disponivel em: <http://www.rwi-essen.de/media/content/pages/ publikationen/ruhr-economic-papers/rep_17_700.pdf>. Acesso em: 10 jul. 2017.

43 BURRELL, Alison et al. Potential EU-Mercosur free trade agreement. impact assessment - Main results. Brussels: Publications Office of the European Union, 2011. v. 1. Disponível em: <http://publications.jrc. ec.europa.eu/repository/handle/JRC67394>. Acesso em: 10 jul. 2017.

44 II modello CGE stimola effetti in tutta l'economia con cambiamenti delle politiche commerciali coinvolgendo tutti i settori dei due blocchi regionali. Cfr.: BURRELL, Alison et al. Potential EU-Mercosur free trade agreement: impact assessment - Main results. Brussels: Publications Office of the European Union, 2011. v. 1. Disponível em: <http://publications.jrc.ec.europa.eu/repository/handle/JRC67394>. Acesso em: 10 jul. 2017. 


\section{II tema dell'immigrazione nella campagna referendaria UK: un problema futuro o già attuale peril Mercosul?}

Nei mesi antecedenti la celebrazione del referendum la campagna politicomediatica nel Regno Unito è stata fortemente caratterizzata da una specifica sovraesposizione del tema dell'immigrazione come voce pretensivamente condizionante la scelta fondamentale sul se aderire o meno alla richiesta a favore o contro l'uscita del Regno Unito dall'Unione europea. L'argomento immigrazione è stato, altresì, fortemente strumentalizzato, tanto da lasciar spesso deliberatamente confondere il tema dell'afflusso nel Regno Unito di immigrati extra-comunitari ed extra-europei, compresi i rifugiati ed i richiedenti asilo, con il ben diverso problema dei cittadini dell'UE di provenienza da altri Paesi membri e la libertà di circolazione e stabilimento all'interno del mercato unico di questi ultimi, in un quadro di denunciata crisi del processo di integrazione e dell'Unione europea. ${ }^{45}$ Immigrazione "interna", libertà di circolazione dei cittadini e dei lavoratori, diritti connessi allo status di cittadinanza europea sono stati, in sostanza, al centro del dibattito pubblico per diversi mesi. In particolare, ed oggetto ora della nostra attenzione, forti tensioni si sono manifestate in riferimento ai diritti dei cittadini europei, non nazionali, legittimamente residenti nel Regno Unito, soprattutto i diritti sociali ed i diritti connessi al rapporto di lavoro, ${ }^{46}$ laddove veniva posto al centro della discussione il costo delle relative prestazioni a carico dell'amministrazione pubblica; denunciando addirittura una sorta di messa in competizione dei cittadini britannici, da un lato, ed europei immigrati, dall'altro, per la provvista di tali prestazioni.

Che si tratti di un tema all'attenzione degli sviluppi dell'ordinamento dell'Unione europea è cosa, del resto, nota da tempo. Le tensioni in merito ai diritti sociali dei cittadini europei residenti in altri Paesi dell'Unione, diversi dal proprio, avendo generato infatti una disciplina più restrittiva ad opera del diritto dell'UE, ${ }^{47}$

45 ASHCROFT, Richard; BEVIR, Mark. Pluralism, national identity and citizenship: Britain after Brexit. The Political Quarterly, v. 87, n. 3, p. 355-359, 2016. p. 355 ss.

46 Sulle pesanti tensioni generate dai più recenti sviluppi della giurisprudenza della CGUE in materia di diritto del lavoro rinviamo alla informata riflessione critica di GIUBBONI, S. Libertà d'impresa e diritto del lavoro nell'Unione europea. Costituzionalismo.it, 7 febbraio 2017. Disponível em: <http://www. costituzionalismo.it/articoli/598/>. Acesso em: 10 jul. 2017. Sulle tensioni tra integrazione dei mercati e garanzia dei diritti sociali già GIUBBONI, Stefano. Social rights and market freedom in the european Constitution. A Labour Law Perspective. Translated by Rita Inston. Cambridge: Cambridge University Press, 2005.

47 A partire dalla Dir. del Parlamento e del Consiglio 2004/38/EC del 29 aprile 2004, con scadenza dal 30 aprile 2006. 
come vedremo determinando a cascata significativi interventi da parte della stessa Corte di Giustizia dell'UE (CGUE). ${ }^{48}$

Nell'ottica funzionalistica in cui muove, fin dalle origini, il diritto comunitario, e successivamente il diritto dell'UE, le libertà di circolazione all'interno del mercato unico sono state abitualmente considerate piuttosto come strumenti per la costruzione del mercato che non come diritti individuali di cittadinanza. ${ }^{49}$ L'obiettivo essendo quello di implementare la c.d. competitività del mercato interno europeo al cospetto dei mercati globali. A fronte di tale prospettiva di sviluppo, pertanto, allorché gli effetti della crisi finanziaria avviatasi a far data dal 2008 cominciarono a manifestarsi anche nell'economia reale, i tagli al servizio delle prestazioni sociali dei cittadini che diversi Stati membri furono costretti ad assumere per ragioni di sostenibilità dei propri bilanci pubblici hanno rapidamente condotto ad importanti tensioni di sistema, in considerazione della competizione da parte degli stranieri aventi diritto alle medesime prestazioni; stranieri, ma pur sempre cittadini europei.

Questi processi di qualificazione delle vicende evolutive della crisi finanziaria come aggravate dall'afflusso di immigrati cittadini europei in relazione ai presunti maggiori costi delle prestazioni sociali hanno assunto una ulteriore rilevanza a seguito degli interventi della giurisprudenza della Corte di giustizia, che ha in più riprese sostenuto e garantito le prestazioni connesse ai diritti sociali come diritti individuali di cittadinanza, andando pertanto oltre lo schema funzionalistico, con ciò generando però conflitti interni agli stessi sistemi politici nazionali. Un caselaw, seppur occasionalmente più rights-oriented che non market-oriented, ${ }^{50}$ più aperto alle istanze pretensive degli individui potendo comportare un implemento dei costi di tali prestazioni a carico delle amministrazioni statali. In alcuni momenti concorrendo così a determinare forti contrasti ideologicamente forieri di conflitti animati da istanze nazionalistiche laddove alla riduzione della spesa sociale ad opera della legislazione statale per fronteggiare la crisi, con effetti gravanti su tutti i cittadini, abbiano potuto far da contraltare decisioni giudiziarie, anche di giudici nazionali, di riconoscimento di diritti a tali prestazioni a vantaggio di cittadini stranieri, ma europei legittimamente residenti. Ciò tanto in riferimento

$48 \mathrm{Si}$ veda il bel saggio di TRYFONIDOU, A. The impact of Union Citizenship on the EU's market freedoms. Oxford-Portland: Hart Publishing, 2016.

49 TRYFONIDOU, A. The impact of Union Citizenship on the EU's market freedoms. Oxford-Portland: Hart Publishing, 2016, spec. p. 58, p. 66 ss., p. 79 ss., p. 91 ss., p. 118 ss., p. 155 ss. Per il diritto del lavoro europeo, esemplarmente, GIUBBONI, Stefano. Libertà d'impresa e diritto del lavoro nell'Unione europea. Costituzionalismo.it, 7 febbraio 2017, spec. §6, ma passim. Disponível em: <http://www. costituzionalismo.it/articoli/598/>. Acesso em: 10 jul. 2017.

50 Rinvio ancora all'analisi di TRYFONIDOU, A. The impact of Union Citizenship on the EU's market freedoms. Oxford-Portland: Hart Publishing, 2016. p. 116 s. 
specifico ai tagli di determinate prestazioni, quanto all'introduzione di elementi di condizionamento per la loro fruizione.

Così, ad esempio, ha provocato un acceso dibattito, significativamente rianimatosi proprio in occasione della campagna referendaria nel Regno Unito, il sistema di condizionalità introdotto ad opera delle Housing Benefit (Amendment) Regulations del $2012^{51}$ che ha sottoposto a riduzioni quantitative percentuali gli assegni erogati agli assistiti, in proporzione al numero di vani "in sovrannumero", rispetto alle stime dei bisogni familiari, delle abitazioni dei beneficiari di prestazioni assistenziali. Attraverso una stima del potenziale reddito della famiglia nell'ipotesi di affitto di tali vani, infatti, il provvedimento ha assunto tale situazione di fatto come concorrente alla determinazione dei limiti reddituali limitativi - o addirittura ostativi - delle prestazioni sociali reclamate dai suoi componenti. A fronte di queste significative riduzioni dei livelli di prestazione sociale, generatrici di un ricco ed articolato contenzioso giudiziario in relazione all'utilizzo degli spazi abitativi privati, in quanto considerati "eccedenti" rispetto alle necessità familiari in ordine al numero degli effettivi residenti, ${ }^{52}$ il riconoscimento all'opposto della garanzia di analoghe prestazioni a vantaggio di cittadini europei non nazionali bisognosi, ha spesso purtroppo concorso a generare un clima di risentimento ed ostilità, facilmente strumentalizzabile in sede di campagna referendaria a favore dell'uscita del Regno Unito dall'UE (leave). La classica rivisitazione dei luoghi polemici tipici della c.d. "guerra tra poveri", alimentata dalla presunta competizione tra cittadini e stranieri nel concorso per la fruizione delle prestazioni sociali.

Narrazione polemica certamente agevolata dalle conseguenze della crisi economica. Insieme alla contrazione delle risorse pubbliche destinate al finanziamento della spesa sociale, essa sta infatti ormai da tempo caratterizzando i conflitti politici - soprattutto nel corso delle campagne elettorali - laddove per la prima volta a far data dagli anni ‘50 la questione sociale, così come gli effetti della politica monetaria e delle politiche economiche, vengono spesso ridotte ad argomento di sbrigative polemiche politiche addirittura contrapponendo ideologicamente tra loro, gli uni agli altri, gli stessi Stati membri. Così portando al centro del dibattito la questione dell'eguaglianza tra cittadini europei, nazionali e stranieri, nell'ambito della proiezione territoriale e della responsabilità politica dei

51 STATUTORY Instrument n. 3040 del 3 dicembre 2012. Social Security. Legislation.gov.uk. Disponível em: <http://www.legislation.gov.uk/uksi/2012/3040/contents/made>. Acesso em: 27 jun. 2017.

52 Si veda di recente, a titolo di esempio, la decisione della Supreme Court UKSC 58 [2016] nel caso $R$ (Carmichael and Rourke) vs. Secretary of State for Work and Pensions, consultabile all'indirizzo <http:// www.bailii.org/uk/cases/UKSC/2016/58.html>. Per un commento della decisione e la citazione di diversi interessanti precedenti si v. RAINE, T. The value of Article 14 ECHR: The Supreme Court and the "Bedroom Tax" U.K. Const. Law Blog, 28 ${ }^{\text {th }}$ Nov. 2016. Available at: <https://ukconstitutionallaw.org/2016/11/28/ thomas-raine-the-value-of-article-14-echr-the-supreme-court-and-the-bedroom-tax//>. 
singoli Stati membri. Quale ulteriore effetto della crisi di tenuta delle ragioni fondative dell'Unione europea, portando ad emersione una nuova questione polemica: da un lato persone che si ingegnano nello strumentalizzare le occasioni offerte dal diritto di libera circolazione sul territorio comune europeo in cerca dei benefici delle prestazioni sociali nei sistemi più generosi o più agevolmente aggirabili (si pensi alle ipotesi dei falsi matrimoni a fini di ricongiungimento familiare). Dall'altro lato l'implementazione di politiche pubbliche e meccanismi istituzionali finalizzati ad evitarlo, a volte proprio limitando le possibilità di accesso a determinate prestazioni sociali, contenuto di diritti riconosciuti anche agli stranieri in quanto cittadini europei. Con il forte rischio di discriminazioni in base alla nazionalità, seppur motivate da ragioni oggettive.

Così, guardando alla giurisprudenza più recente della Corte di giustizia sulla libertà di circolazione e di stabilimento dei cittadini lavoratori all'interno del mercato unico, ricaviamo ulteriori importanti segnali di tendenza circa il tenore dei contenuti che la attuale disciplina dei diritti a prestazione sociale posta dal diritto dell'UE riflette sullo stato di cittadinanza europea. In particolare appare significativa la giurisprudenza relativa alla citata direttiva 2004/38/EC ${ }^{53}$ nei casi dei cd. lavoratori economicamente inattivi, quali i disoccupati al termine dei periodi di efficacia delle misure di sostegno al reddito e quindi divenuti bisognosi di prestazioni di assistenza sociale; i pensionati al minimo economicamente non autosufficienti; i nuclei familiari privi di copertura assicurativa sanitaria; etc. Gli Stati membri cominciano sempre più di frequente a vedersi riconosciuta dall'UE la facoltà di negare il diritto di residenza a cittadini europei di altri Stati membri, privi di risorse economiche sufficienti, tali da assicurare che per loro e le loro famiglie non si determinino le condizioni per la pretesa erogazione di prestazioni assistenziali, sanitarie, scolastiche o altre agevolazioni tariffarie per la fruizione di servizi pubblici che finiscano con il pesare sui sistemi di welfare del Paese ospitante aggravandone i relativi costi. ${ }^{54}$ Tutti sintomi di un mutato clima generale

53 Attuata in Italia con d. I.vo 6 febbraio 2017, n. 30; in Spagna ad opera del Real Decreto 16 febbraio 2007, n. 240 "sobre entrada, libre circulación y residencia en España de ciudadanos de los Estados miembros de la Unión Europea y de otros Estados parte en el Acuerdo sobre el Espacio Económico Europeo", provvedimenti ai quali si rinvia per la verifica dell'analogo tenore nel senso di cui nel testo. La questione è ben analizzata nel saggio di CAVASINO, E. Diritti europei e non nazionali? Sullo statuto giuridico del migrante: il nodo degli standards sovranazionali comuni. Revista de Derecho de la Unión Europea, n. 3031, p. 291-312, 2016. p. 299 ss., p. 303 ss. Ma si veda, altresì, la sentenza del Tribunale costituzionale spagnolo n. 139/2016, del 21 luglio 2016, pto 10 della Parte II. Fundamentos jurídicos, in Boletín Oficial del Estado, Sección del Tribunal Constitucional, n. 196 del 15 agosto 2016, 60502 ss., spec. 60532.

54 Si vedano, tra le tante, le sentenze CGUE, C-333713, Grande Camera, 11 novembre 2014, Elisabetta Dano e Florin Dano vs. Jobcenter Leipzig; CGUE, C-67/14, Grande Camera, 15 settembre 2015, caso Jobcenter Berlin Neukölln vs. Alimanovic N., S., V., V.; CGUE, C-308/14, Prima Sezione, 14 giugno 2016, caso Commissione europea vs. Regno Unito. Sul punto, altresì, TRYFONIDOU, A. The impact of Union Citizenship on the EU's market freedoms. Oxford-Portland: Hart Publishing, 2016. p. 30 ss., p. 79 ss., 
in riferimento alle modalità di esercizio della libertà di circolazione delle persone, il cui regime giuridico in Europa ${ }^{55}$ sta finendo con il rendere del tutto plausibile proprio quella guerra tra poveri i cui drammatici segnali abbiamo ritenuto di individuare nei discorsi animati dalla campagna referendaria per la Brexit ma che, purtroppo, hanno assunto un ruolo anche nella dimensione ufficiale delle relazioni diplomatiche tra i governi degli Stati membri, nei reciproci rapporti ed in seno alle istituzioni dell'Unione europea.

La sfida attuale per gli scienziati sociali, politici, per gli economisti, è rappresentata dal vedere come tale situazione possa ripetersi in altri contesti regionali, in altri sistemi istituzionali di integrazione di mercati.

\section{Considerazioni finali}

II tema al quale solo accenniamo per fare ad esso rinvio come possibile sviluppo di ulteriori riflessioni è pertanto rappresentato dal complicato nesso tra diritto di matrice giurisprudenziale dei diritti fondamentali, nel sistema inglese di chiaro radicamento nel common law, e diritto europeo dei diritti fondamentali, anch'esso di matrice giurisprudenziale ma di derivazione esterna, prodotto dal case-law della Corte di giustizia e della Corte europea dei diritti dell'uomo. Nel quale si intrecciano le due distinte questioni del rapporto tra common law e statute law prodotto della sovranità parlamentare in tema di diritti fondamentali, da un lato; e la questione della pretesa supremazia del diritto europeo sul principio della sovranità parlamentare, dall'altro. Ma quel che qui si rileva, seppur su tale sfondo problematico, è altro. Vale a dire l'utilizzo strumentale della questione immigrazione come problema, proprio in virtù del paventato rischio che le giurisdizioni "pretendano" di tutelare i diritti degli stranieri, anche europei, anche contro le scelte "sovrane" di una classe politica che voglia, al contrario, metterne in discussione i fondamenti sulla spinta di un presunto mandato popolare, provocato ad arte dalla strumentale deriva ideologica assunta quale discorso pubblico da un approccio populistico foriero purtroppo di più forte consenso. Un circolo vizioso, quindi, ed una deriva contraria all'espansione delle tutele.

p.197 s.; CAVASINO, E. Diritti europei e non nazionali? Sullo statuto giuridico del migrante: il nodo degli standards sovranazionali comuni. Revista de Derecho de la Unión Europea, n. 30-31, p. 291-312, 2016.

55 Esemplarmente, per la Spagna, si veda il Rapporto del Gobierno de España, Secretaría de Estado de la Seguridad Social (GONZÁLEZ ORTEGA, Santiago (Org.). La protección social de los trabajadores extranjeros. [s.l.]: Gibierno de España. spec. p. 33 ss., p. 39 ss. Disponível em: <http://www.seg-social.es/prdi00/ groups/public/documents/binario/113305.pdf>. Acesso em: 27 jul. 2017). 
II che ci porta sull'ultimo punto rilevante ai fini di queste considerazioni. La questione dell'eguaglianza. Quella stessa eguaglianza che, pur nella sua declinazione formale, come divieto di discriminazione in base alla nazionalità, costituisce il fondamento della libertà di circolazione e soggiorno garantita ai lavoratori dai trattati europei. Libertà di circolazione delle persone, appunto, sempre più trattate dal diritto europeo alla stregua della libertà di commercio, come libera circolazione delle merci, cioè. Anche se tale diritto riguarda in verità, come detto, le persone, non le cose.

È noto che l'impatto della recente crisi economico-finanziaria non ha prodotto identici effetti nei diversi Paesi membri del mercato comune. Germania, Austria, Paesi Bassi e Belgio hanno visto il tasso di occupazione calare soltanto marginalmente, mentre in Spagna, Italia, Irlanda, Grecia e Portogallo i livelli di disoccupazione raggiunti si dimostrano significativamente molto gravi. Dai dati statistici emerge con chiarezza che la crisi ha prodotto, nella sostanza, vincitori e vinti, tra le economie dei diversi Stati, e all'interno di questi tra parti e parti della popolazione. Così come hanno prodotto fortissime diseguaglianze tanto le ultime recenti fasi del processo di integrazione europea, soprattutto a partire dall'entrata in vigore della moneta unica, anche se non certo a causa dell'Euro come da alcuni incautamente sostenuto, quanto i processi di globalizzazione. Diseguaglianze che hanno un impatto profondo sul piano economico, sociale e quindi politico. Guardando alla cittadinanza europea attraverso lo spettro della giurisprudenza della Corte di giustizia, ${ }^{56}$ quindi, si comprende come possa essere accaduto che le aperture a favore dei diritti sociali come diritti individuali funzionali ai diritti di cittadinanza, e non più soltanto ai processi di integrazione dei mercati, come più sopra ricordato, possano aver generato un diffuso sentimento di ostilità verso gli stranieri-cittadini europei. Anche questi aventi diritto a tali prestazioni, e quindi considerati competitori indesiderati da parte di chi vedeva così ridursi le proprie chances di accesso ai processi redistributivi di ricchezza, sempre più difficoltosi in sé già a causa della crisi economica.

Un quadro problematico divisivo lungo l'asse delle diseguaglianze tra cittadino e straniero anche se cittadino europeo, in una parola e di nuovo la questione "immigrazione". Questione che riporta in auge l'idea di una lotta contro i diritti individuali (degli altri), soprattutto diritti di eguaglianza e prestazioni sociali, lotta da assumere così facilmente come strumento per il rafforzamento di una rinnovata identità nazionale, anzi identità nazionali, al plurale, foriere di rinnovati

56 GIUBBONI, Stefano. Libertà d'impresa e diritto del lavoro nell'Unione europea. Costituzionalismo.it, 7 febbraio 2017. Disponível em: <http://www.costituzionalismo.it/articoli/598/>. Acesso em: 10 jul. 2017, spec. cit., §6. 
conflitti tra i popoli. Un ritorno alla sovranità nazionale nella forma più retrograda, in quanto declinata quale attributo del "popolo", del suo governo, non più della costituzione e dei diritti. Guardando in giro per le cancellerie europee, sotto le pressioni populistiche dei partiti antieuropei, infatti, ad essere messa a tema è una rinnovata dottrina espansiva dei poteri dei governi nazionali da costruire attraverso la polemica contrapposizione tra questi ultimi ed i sistemi istituzionali europeo e sovranazionale. Un'espansione fondata sulla riduzione delle prestazioni sociali in generale e, comunque, a vantaggio dei soli cittadini nazionali; il contrasto alla libertà di circolazione anche se esercitata nelle forme legali; una sempre più diffusa e mal motivata critica nei confronti dei sistemi di giustizia costituzionale e di protezione giudiziaria dei diritti fondamentali, compresi ovviamente i sistemi di protezione regionale ed internazionale dei diritti nei confronti dei poteri statali sovrani; la concentrazione dei poteri in capo al governo, schiacciando sempre più sulla volontà di quest'ultimo il ruolo del Parlamento.

Non tutto comprensibile, non tutto fisiologicamente conseguente alle cause di crisi politica e di effettività degli ordinamenti costituzionali che pur si sono più sopra segnalate, ma con fenomeni distruttivi senz'altro accentuati dall'uso del referendum o degli appelli al popolo quale strumento di lotta contro il processo di integrazione europea, le istituzioni dell'UE e le altre organizzazioni sovranazionali a vocazione costituzionale, come ad esempio, sempre in Europa la Convenzione Europea dei Diritti dell'Uomo e delle Libertà fondamentali (CEDU). Processi all'esito dei quali resterà soltanto da chiedersi, infine, cosa ne potrà mai essere dei diritti fondamentali e di cittadinanza, dei diritti economico-sociali e dei diritti dei lavoratori nel nuovo contesto costituzionale che si va così definendo. II tutto in un quadro di rinnovata conflittualità tra Stati, nei reciproci rapporti ed in seno alle istituzioni europee, che sembra riportare l'Europa indietro di esatti cento anni.

È possibile, in senso contrario, che in un prossimo futuro i britannici decidano che la decisione adottata col referendum non fosse la più appropriata; pensando ad una società mondiale, dovranno anch'essi fare i conti con un certo numero di Paesi che ospitano oltre un milione di cittadini britannici in altre nazioni e anche con gli europei che vivono nel Regno Unito. E le ripercussioni non sono solo finanziarie: il Regno Unito, anche se è uno dei Paesi più ricchi, non può vivere in isolamento né aspettare che una situazione di grande sviluppo industriale possa essere recuperata immediatamente. La preoccupazione principale dei più anziani - e per questo si ritiene che questi possano avere votato in massa per lasciare I'Unione europea - è rappresentata dalla disoccupazione, che viene collegata all'idea che gli stranieri rappresentino la principale causa della crisi dell'occupazione dei cittadini britannici. Possiamo prevedere che l'Unione europea possa superare tale crisi, inusuale ed inattesa, e che in seguito la vita in comune sul pianeta Terra 
possa dipendere in misura molto maggiore dall'integrazione, piuttosto che non dalla disintegrazione in atto?

\section{Referendo do Reino Unido sobre Brexit e seu impacto no Mercosul na perspectiva de direitos sociais}

Resumo: Tendo em vista as temáticas polêmicas ocorridas na campanha do Reino Unido em junho de 2016 sobre o Brexit, um sábio reflete sobre as transformações do tempo no presente, no direito da UE e na jurisprudência da Corte de Justiça, assim como em alguns ordenamentos dos membros de Estado, em relação aos direitos e prestações sociais dos cidadãos europeus presentes nos países membros que não Reino Unido e ao possível impacto de tal fenômeno nos outros sistemas integrados de mercado, como o Mercosul. 0 quadro que emerge induz a aprofundar mais reflexões sobre as causas da crise de legitimação atual dos sistemas de integração política e econômica, como o processo de integração europeia, cujo cedimento é gravemente acelerado daquele em discussão dos direitos sociais atingidos de uma noção mais forte de cidadania europeia; e como em outras realidades institucionais e sociais, particularmente o Mercosul.

Palavras-chave: Brexit. Mercosul. Direitos sociais. União Europeia.

Sumário: 1 Premissa: 0 direito na sociedade contemporânea -20 referendum sobre o BREXIT e os seus reflexos no Mercosul - 30 tema da imigração na campanha do referendum da UK: um problema futuro ou atual para o MERCOSUL? - 4 Considerações finais - Referências

\section{United Kingdom referendum on Brexit and its impact on Mercosur in the perspective of social rights}

Abstract: Owing to the polemic occurred at the UK in june 2016 about Brexit, some wise reflexion about the transformation of the time at the present, to the right of the EU and the jurisprudence of the court of justice so as in some orders of the countries, about the rights and social prestations of the europeans citziens members of the other countries (not UK) and the possible impact of such phenomenon at the others systems of politics and market such as Mercosul. The situations induce to profound the reflexion of such causes of atual legitimation crisis of the systems of politics and economics, as the process of european integration, which subsidence is severely accelerated from the discussion of the social rights stunned by a strong meaning of european citzien; such as in other institucional and social realities, like Mercosul.

Keywords: Brexit. Mercosul. Social rights. European Union.

Summary: 1 A premise: law in contemporary society - $\mathbf{2}$ The referendum on BREXIT and its results on Mercosur - $\mathbf{3}$ The theme of immigration in the UK referendum campaign: a future or current problem for MERCOSUR? - 4 Final considerations - References

\section{Bibliografia}

AMORIM, Celso. A terra treme no Mercosul. Carta Capital, São Paulo, 1ํago. 2016. Disponível em: <www.cartacapital.com.br/politica/a-terra-treme-no-mercosul>. Acesso em: 27 jun. 2017.

ASHCROFT, Richard; BEVIR, Mark. Pluralism, national identity and citizenship: Britain after Brexit. The Political Quarterly, v. 87, n. 3, p. 355-359, 2016. 
AYRAL, Michel. Le marché intérieur de l'Union européenne: les règles du jeu. Paris: Le Documentation Française, 1998.

BADR, Eid. O direito comunitário e o Mercosul à luz da Constituição Federal brasileira. Curitiba: CRV, 2011.

BELKE, Ansgar; GROS, Daniel. The economic impact of Brexit: evidence from modelling free trade agreements. Brussels, 2017. Disponivel em: <http://www.rwi-essen.de/media/ content/pages/publikationen/ruhr-economic-papers/rep_17_700.pdf>. Acesso em: 10 jul. 2017.

BILANCIA, Francesco et al. Le forme della democrazia contemporanea e il germe della sua autodistruzione. In: GIANELLI, A. et al. (A cura di). Democrazia. Storia e crisi di una forma politica. Napoli: Editoriale Scientifica, 2013.

BILANCIA, Francesco. Sovranità. Rivista AIC, n. 3, 2017.

BRADLEY, A. Pressure on a Historical Constitution: the Brexit decision in the UK's Supreme Court. Dir.pubbl., n. 1, 2017.

BRASIL, Argentina, Paraguai e Uruguai suspendem Venezuela do Mercosul. Revista Valor Econômico, 1ํjan. 2016. Disponível em: <http://www.valor.com.br/brasil/4793901/brasilargentina-paraguai-e-uruguai-suspendem-venezuela-do-mercosul>. Acesso em: 27 jun. 2017.

BRUNELLI, G. et al. Dal governo democratico-rappresentativo al governo a mera legittimazione popolare. (Brevi riflessioni sui mutamenti di regime). In: BRUNELLI, Giuditta; PUGIOTTO, Andrea; VERONESI, Paolo (A cura di). Scritti in onore di Lorenza Carlassare. Napoli: Jovene, 2009.

BURRELL, Alison et al. Potential EU-Mercosur free trade agreement. impact assessment Main results. Brussels: Publications Office of the European Union, 2011. v. 1. Disponivel em: <http://publications.jrc.ec.europa.eu/repository/handle/JRC67394>. Acesso em: 10 jul. 2017.

CAVASINO, E. Diritti europei e non nazionali? Sullo statuto giuridico del migrante: il nodo degli standards sovranazionali comuni. Revista de Derecho de la Unión Europea, n. 30-31, p. 291-312, 2016.

CORE Europe to the rescue: a conversation with Jürgen Habermas about Brexit and the EU crisis. Social Europe, 12 jul. 2016. Disponível em: <https://www.socialeurope.eu/coreeurope-to-the-rescue>. Acesso em: 27 jun. 2017.

DíAZ LABRANO, Roberto Ruíz. La integración y las constituiciones nacionales de los Estados Parte del Mercosur. In: KONRAD ADENAUER STIFTUNG. Anuario de Derecho Constitucional. Buenos Aires: CIEDLA, Konrad-Adenauer-Stiftung, 1999.

EUROPEAN Union (Notification of Withdrawal) Act 2017. Legislation.gov.uk. Disponível em: <http://www.legislation.gov.uk/ukpga/2017/9/enacted>. Acesso em: 27 jun. 2017.

FABBRINI, F. Brexit according to the UK Supreme Court: the Miller Judgement. Centro Studi Sul Federalismo, n. 100, 27 gennaio 2017.

FINSBURY Park attack: what we know so far. BBC, 19 jun. 2017. Disponivel em: <http:// www.bbc.com/news/uk-40323769>. Acesso em: 27 jun. 2017. 
FOREIGN direct investment statistics. Eurostat Statistics Explained, abril 2017. Disponível em: $<$ http://ec.europa.eu/eurostat/statistics-explained/index.php/Foreign_direct_investment_ statistics>. Acesso em: 10 jul. 2017.

GALLIGAN, D. The Constitution in Crisis. Oxford: Wolfson College, 2016.

GEUSS, Raymond. Beni pubblici bene privato: origine e significato di una distinzione. Roma: Donzelli Editore, 2005.

GIRALDI, Renata. Paraguai cogita referendo para definir permanência no Mercosul. Carta Capital, São Paulo, 15 ago. 2012. Disponível em: <https://www.cartacapital.com.br/ internacional/paraguai-cogita-referendo-para-definir-permanencia-no-mercosul>. Acesso em: 28 jun. 2017.

GIUBBONI, Stefano. Libertà d'impresa e diritto del Iavoro nell'Unione europea. Costituzionalismo.it, 7 febbraio 2017. Disponivel em: <http://www.costituzionalismo.it/ articoli/598/>. Acesso em: 10 jul. 2017.

GIUBBONI, Stefano. Social rights and market freedom in the european Constitution. A Labour Law Perspective. Translated by Rita Inston. Cambridge: Cambridge University Press, 2005.

GONZÁLEZ ORTEGA, Santiago (Org.). La protección social de los trabajadores extranjeros. [s.l.]: Gibierno de España. Disponível em: <http://www.seg-social.es/prdiO0/groups/public/ documents/binario/113305.pdf>. Acesso em: 27 jul. 2017.

HÁ 'otimismo realista' com acordo entre Mercosul e UE, diz secretário. Revista Época Negócios, 6 jul. 2017. Disponível em: <http://epocanegocios.globo.com/Mundo/ noticia/2017/07/ha-otimismo-realista-com-acordo-entre-mercosul-e-ue-diz-secretario.html>. Acesso em: 10 jul. 2017.

HARLOW, Carol. The limping legitimacy of EU Lawmarking: a barrier to integration. Eur Papers, 2016.

HOUSE OF COMMONS - EXITING THE EUROPEAN UNION COMMITTEE. The Process for exiting the European Union and the Government's negotiating objectives. 2017. Disponivel em: <https://publications.parliament.uk/pa/cm201617/cmselect/cmexeu/815/815.pdf>. Acesso em: 27 jul. 2017.

JAEGER JUNIOR, Augusto. Metodologia jurídica europeia e mercosulista: considerações fundamentais. Revista da Secretaria do Tribunal Permanente de Revisão do Mercosul, Assunção, n. 3, mar. 2014.

JAEGER JÚNIOR, Augusto; DIZ, Jamile Bergamaschine Mata. Por uma teoria jurídica da integração regional: a inter-relação direito interno, direito internacional público e direito da integração. Revista de Direito Internacional, Brasília, v. 12, n. 2, p. 138-158, 2015.

JHERING, R. von.; PIOVANI, P. (A cura di). La lotta per il diritto. Bari: Laterza, 1960.

KING, J. What next? Legislative authority for triggering art. 50. UK Constitutional Law Association. Disponivel em: <https://ukconstitutionallaw.org/2017/01/24/jeff-king-whatnext-legislative-authority-for-triggering-article-50-2/>. Acesso em: 27 jun. 2017.

LEYLAND, P. Brexit and the UK: charting the constitutional and legal obstacles. Istituzioni del Federalismo, 2017. In corso di pubblicazione. 
LORENZETTI, Ricardo. Sistema Jurídico del Mercosur. In: ARAUJO, Nadia de; MARQUES, Claudia Lima (Org.). O novo direito internacional: estudos em homenagem a Erik Jayme. Rio de Janeiro: Renovar, 2005.

LUCIANI, M. Art. 75. II referendum abrogativo. In: BRANCA, Giuseppe (A cura di). Commentario della Costituzione. Bologna: N. Zanichelli; Roma: Soc. Ed. del Foro Italiano, 2005.

LUHMANN, Niklas. Sociologia del rischio. Milano: Bruno Mondadori, 1996.

MERCOSUL suspende Paraguai e anuncia adesão da Venezuela. G1, 29 jun. 2012. Disponível em: <http://g1.globo.com/mundo/noticia/2012/06/mercosul-suspende-paraguai-e-anunciaadesao-da-venezuela.html>. Acesso em: 27 jun. 2017.

MEZZANOTTE, C.; NANIA, R. Referendum e forma di governo in Italia. Dem.dir., 1981.

NEGOCIADOR quer fechar ponto chave de acordo UE-Mercosul esse ano. Revista Exame, 6 jul. 2017. Disponivel em: <http://exame.abril.com.br/economia/negociador-quer-fecharponto-chave-de-acordo-ue-mercosul-esse-ano/>. Acesso em: 10 jul. 2017.

OLIVAR JIMENEZ, Martha Lucía. La compreensión de la noción de derecho comunitario para una verdadera integración en el Cono Sur. In: BASSO, Maristela (Org.). Mercosul: seus efeitos jurídicos, econômicos e poíticos nos estados-membros. Porto Alegre: Livraria do Advogado, 1997.

OLIVEIRA, Odete Maria. União Europeia: processos de integração e mutação. Curitiba: Juruá, 2001.

OLIVEIRA, R.; MARREIRO, F. UE e Mercosul correm para fechar acordo antes do avanço dos nacionalismos. Jornal El País, 6 maio 2017. Disponivel em: <https://brasil.elpais.com/ brasil/2017/05/05/internacional/1494017908_326426.html>. Acesso em: 10 jul. 2017.

OSSERVATORIO sulla Brexit 25 maggio - 21 giugno 2017. Federalismi.it. Disponível em: <http://www.federalismi.it/nv14/articolo-documento.cfm?Artid=32932>. Acesso em: 27 jul. 2017.

PROTOCOLO de Montevideu Sobre o Compromisso com a Democracia no Mercosul. Mercosul. Disponível em: <dai-mre.serpro.gov.br/atos-internacionais/multilaterais/protocolo-demontevideu-sobre-compromisso-com-a-democracia-no-mercosul-ushuaia-ii-firmado-por-ocasiaoda-xlii-reuniao-do-cmc-e-cupula-de-presidentes-do-mercosul-e-estados-associados/>. Acesso em: 27 jun. 2017.

RAINE, T. The value of Article 14 ECHR: The Supreme Court and the "Bedroom Tax" U.K. Const. Law Blog, 28 ${ }^{\text {th }}$ Nov. 2016. Available at: <https://ukconstitutionallaw.org/2016/11/28/ thomas-raine-the-value-of-article-14-echr-the-supreme-court-and-the-bedroom-tax//>.

RESTA, Eligio. L'inimicizia, l'umanità, le guerre. Sociologia del diritto, n. 1, 2001.

SAIBA mais sobre o Mercosul. Mercosul. Disponível em: <http://www.mercosul.gov.br/ saiba-mais-sobre-o-mercosul\#DADOSGERAIS>. Acesso em: 27 jun. 2017.

SGRÒ, F. II caso "Brexit": qualche considerazione sulla sovranità parlamentare e sul sistema delle fonti nell'ordinamento costituzionale britannico dopo la sentenza della Supreme Court of the United Kingdom. Federalismi.it, n. 5, 2017. 
SOARES FILHO, José. Mercosul: surgimento, estrutura, direitos sociais, relação com a Unasul, perspectivas de sua evolução. Revista CEJ, Brasília, ano XIII, n. 46, jul./set. 2009.

STATUTORY Instrument n. 3040 del 3 dicembre 2012. Social Security. Legislation.gov.uk. Disponivel em: <http://www.legislation.gov.uk/uksi/2012/3040/contents/made>. Acesso em: 27 jun. 2017.

THE United Kingdom's exit from and new partnership with the European Union. Gov.uk, febbraio 2017. Disponivel em: <https://www.gov.uk/government/publications/the-united-kingdomsexit-from-and-new-partnership-with-the-european-union-white-paper>. Acesso em: 27 jul. 2017.

TRYFONIDOU, A. The impact of Union Citizenship on the EU's market freedoms. OxfordPortland: Hart Publishing, 2016.

UE e Mercosul trocam ofertas tarifárias por acordo comercial. G1, maio 2016. Disponível em: <http://g1.globo.com/economia/noticia/2016/05/ue-e-mercosul-trocam-ofertas-tarifariaspor-acordo-comercial.html>. Acesso em: 27 jul. 2017.

UKSC (5) 2017 del 24 gennaio 2017. The Supreme Court, 2017. Disponivel em: <https:// www.supremecourt.uk/cases/docs/uksc-2016-0196-judgment.pdf>. Acesso em: 27 jul. 2017.

WADSWORTH, Jonathan et al. Brexit and the Impact of Immigration on the UK. London: Centre for Economic Performance; London School of Economics and Political Science. Disponivel em: <http://cep.Ise.ac.uk/pubs/download/brexit05.pdf>. Acesso em: 27 jun. 2017.

ZOLO, Danilo. Globalizzazione. Una mappa dei problemi. Bari: Laterza, 2009.

Informação bibliográfica deste texto, conforme a NBR 6023:2002 da Associação Brasileira de Normas Técnicas (ABNT):

BILANCIA, Francesco; MARTINI, Sandra Regina. II referendum del Regno Unito sulla Brexit ed il suo impatto sul mercosul nella prospettiva dei diritti sociali. Direitos Fundamentais \& Justiça, Belo Horizonte, ano 11, n. 37, p. 35-63, jul./dez. 2017.

Recebido em: 31.10.2017

Aprovado em: 31.10.2017 (cota convite) 